\title{
GMR
}

\section{Shock wave treatment enhances endothelial proliferation via autocrine vascular endothelial growth factor}

\author{
Y.Z. Peng ${ }^{1 *}$, K. Zheng ${ }^{2 *}$, P. Yang ${ }^{1}$, Y. Wang ${ }^{1}$, R.J. Li ${ }^{1}$, L. Li ${ }^{1}$, J.H. Pan ${ }^{1}$ and T. Guo ${ }^{1}$ \\ 'Department of Cardiology, The First Affiliated Hospital, Kunming Medical University, \\ Kunming, Yunnan, China \\ 2Department of Breast Surgery, The Third Affiliated Hospital, \\ Kunming Medical University, Kunming, Yunnan, China \\ *These authors contributed equally to this study. \\ Corresponding author: T. Guo \\ E-mail: guotao20@hotmail.com
}

Genet. Mol. Res. 14 (4): 19203-19210 (2015)

Received August 23, 2015

Accepted October 10, 2015

Published December 29, 2015

DOI http://dx.doi.org/10.4238/2015.December.29.30

\begin{abstract}
Extracorporeal cardiac shock wave (SW) therapy is an effective, safe, and non-invasive therapeutic strategy for severe coronary artery disease. Shock wave therapy might affect cardiac tissues because of its ability to promote angiogenesis. In this report, we investigated if the up-regulation of vascular endothelial growth factor (VEGF) by SW therapy is involved in cell proliferation in cultured endothelial cells. After human umbilical vein endothelial cells were treated with SW, the expression and secretion of VEGF as well as cell proliferation were analyzed. We also determined the mechanism underlying SW-induced the extracellular signal-regulated kinase (ERK) mitogen-activated protein kinase (MAPK) using western blotting. Our results demonstrated that SW treatment induced VEGF expression in endothelial cells in a hypoxia-inducible factor 1-independent manner. Up-regulation of VEGF expression led to an increase in its concentration in the cultured medium. The autocrine VEGF in the medium activated the ERK MAPK signaling, which in turn
\end{abstract}


enhanced cell proliferation. Therefore, we concluded that VEGF mediates SW application-induced endothelial cell proliferation in a cell-autonomous manner.

Key words: Extracorporeal cardiac shock; Endothelial cell; ERK MAPK; Vascular endothelial growth factor

\section{INTRODUCTION}

Ischemic heart disease is the leading cause of death in the world (Jessup and Brozena, 2003). Narrowing of the coronary vessel diameter, or occlusion of a coronary artery may result in myocardial ischemia and infarction. This process, in turn, leads to heart failure, which is associated with a high level of mortality (Caro et al., 2005). The major therapeutic strategies for the treatment of ischemic heart disease include drug therapy, percutaneous coronary intervention, and coronary artery bypass grafting. However, patients without an indication for coronary intervention or bypass surgery generally show poor prognosis (Jessup and Brozena, 2003). Angiogenesis is one of the most promising therapeutic strategies for severe ischemic heart disease.

Extracorporeal shock wave therapy (ESWT) has been recently introduced as a novel noninvasive option for the treatment of patients with severe coronary artery disease, such as angina pectoris, ischemic heart failure, and systolic dysfunction (Fukumoto et al., 2006; Khattab et al., 2007; Kikuchi et al., 2010; Vasyuk et al., 2010). ESWT has been shown to promote coronary angiogenesis in the ischemic myocardium and improve myocardial function in vivo in a porcine model of chronic myocardial ischemia (Nishida et al., 2004). Meanwhile, ESWT has also been effective in the promotion of angiogenesis in a rabbit model of hindlimb ischemia (Oi et al., 2008). The precise mechanism underlying ESWT-induced angiogenesis remains unknown. In vitro and in vivo experiments reveal that shock wave (SW) treatment significantly up-regulates the expression of vascular endothelial growth factor (VEGF) in cultured human umbilical vein endothelial cells (HUVECs) and the ischemic myocardium (Nishida et al., 2004). These studies raised the possibility of the involvement of VEGF in ESWT-induced angiogenesis. To test this hypothesis, the effect of SW treatment on cell proliferation in HUVECs was investigated. Our results indicated that SW treatment promotes endothelial proliferation by VEGF in a cell-autonomous manner.

\section{MATERIAL AND METHODS}

\section{Cell culture and SW treatment}

Umbilical cord tissues were obtained from 25 healthy pregnant women, who were fulltime patients of the First Affiliated Hospital, Kunming Medical University. The study was approved by the Kunming Medical University Ethics Committee, and conducted according to the approved guidelines. Written informed consent was also obtained from all participants. HUVECs were isolated from the umbilical veins by irrigative digestion. The cells were then cultured in complete endothelial medium (EGM-2 BulletKit; Clonetics, San Diego, CA, USA) supplemented with $2 \%$ fetal bovine serum, and maintained at $37^{\circ} \mathrm{C}$ in a $95 \%$ air and $5 \% \mathrm{CO}_{2}$ atmosphere.

HUVECs at passages 4 and 5 were subjected to SW treatment. HUVECs at $70-80 \%$ confluence were cultured in basal medium (EBM-2; Clonetics), and serum-starved for $12 \mathrm{~h}$. The cells $\left(1 \times 10^{6}\right)$ were then suspended in a $2-\mathrm{mL}$ tube with the medium and treated with 600 impulses 
of $S W$ at an energy flux density of $0.1 \mathrm{~mJ} / \mathrm{mm}^{2}$. The suspended cells were seeded on plates in the same medium for further experimentation.

\section{Proliferation assay}

Following SW treatment, HUVECs were seeded on 96-well plates $\left(1 \times 10^{4}\right.$ cells per well). The cells were washed with PBS after 2 days, and the cell proliferation determined using the 3-(4,5-dimethylthiazol-2-yl)-2,5-diphenyltetrazolium bromide (MTT; Sigma-Aldrich, St. Louis, MO, USA) assay, as previously described (Vucenik et al., 2004).

The effect of autocrine VEGF on HUVEC proliferation was determined by adding antihuman VEGF antibodies (10 ng per well; R\&D Systems, Minneapolis, MN, USA) or isotype control antibodies (mouse IgG, 10 ng per well; Santa Cruz Biotechnology, Santa Cruz, CA, USA) to 96-well plates, after plating of the cells.

\section{Flow cytometry for cell-cycle assay}

Following SW treatment, the HUVECs were seeded on 24-well plates $\left(5 \times 10^{4}\right.$ cells per well) and incubated for $24 \mathrm{~h}$. Subsequently, the cells were washed twice with PBS; the cells were collected following trypsinization and resuspended. DNA Prep ${ }^{T M}$ LPR was added to the cell suspension, and the samples were incubated for $20 \mathrm{~min}$ in the dark. The cell suspension was again incubated with DNA Prep ${ }^{\mathrm{TM}}$ Stain for $20 \mathrm{~min}$. The cell cycle analysis was then performed using a flow cytometer (BD Biosciences, San Jose, CA, USA).

\section{Quantitative real-time polymerase chain reaction (PCR)}

Total RNA was isolated from the HUVECs using TRIzol reagent (Invitrogen, Carlsbad, CA, USA). Random-primed cDNA was obtained from total mRNA by reverse transcription. A realtime PCR assay was performed in the ABI Prism 7300 Sequence Detection System (Applied Biosystems, Foster City, CA, USA), using the SYBR Premix-Ex TagTM (TaKaRa, Dalian, China) according to the manufacturer protocols. The primers used for PCR were: VEGF, 5'-CTT GCT CTC TTA TTT GTA-3' (F); 5'-TAG TAT GTA GAT GTA TAT TGAA-3' (R); and GAPDH, 5'-CAA GGT CAT CCA TGA CAA CTT TG-3' (F); 5'-GTC CAC CAC CCT GTT GCT GTA G-3' (R).

\section{Western blotting}

SW-treated HUVECs were collected by trypsinization. The cells were then lysed using a lysis buffer (BioTeKe, Beijing, China) for $60 \mathrm{~min}$. The supernatant protein $(40 \mu \mathrm{g})$ was loaded to each lane and separated on a $10 \%$ sodium dodecyl sulfate (SDS)-polyacrylamide gel. The proteins were then transferred from the SDS gels to polyvinylidene difluoride membranes, and probed with primary antibodies. The primary antibodies included anti-VEGF (1:2000 dilution), anti-phospho extracellular signal-regulated kinase (ERK) (1:1000 dilution), anti-ERK (1:1000 dilution), anti-HIF$1 \alpha$ (1:2000 dilution), and anti-actin (1:2500 dilution) antibodies (Santa Cruz Biotechnology). The secondary antibody was a peroxidase-coupled anti-rabbit or mouse IgG (1:5000 dilution; Santa Cruz Biotechnology). The membrane was exposed to a Kodak X-Omat film (Kodak, Xiamen, China), and developed for analysis. 


\section{Detection of VEGF secretion}

Following the SW treatment, the HUVECs were seeded on 6 -well plates $\left(1 \times 10^{5}\right.$ cells per well) and incubated for $24 \mathrm{~h}$; subsequently, the medium was collected. The levels of VEGF were determined using commercially available ELISA kits (R\&D Systems, Minneapolis, MN, USA).

\section{Statistical analysis}

All data are reported as means \pm standard deviation (SD) of values from three independent experiments. Statistical differences between the groups were analyzed using the Student paired $t$-test or one-way analysis of variance (ANOVA), followed by a Newman-Keuls post hoc test. P values $<0.05$ were considered to be statistically significant.

\section{RESULTS}

\section{SW treatment promotes HUVEC proliferation.}

Initially, the effect of SW treatment on cell proliferation was evaluated by synchronizing the HUVECs by serum starvation for $24 \mathrm{~h}$. Forty-eight hours after the SW treatment, the cells were subjected to the MTT assay. We observed a substantial increase in the optical density at 540 $\mathrm{nm}\left(\mathrm{OD}_{540}\right)$ of the HUVECs, compared to the control $(\mathrm{P}<0.05$; Figure $1 \mathrm{~A})$. Meanwhile, the DNA content of the cells was analyzed by flow cytometry. A representative flow cytometry experiment, and the percentage of cells in each phase of the cell cycle, is presented in Figure 1B. SW treatment markedly increased the percentage of HUVECs in the $S$ phase from 21 to $33 \%(P<0.001)$, and decreased the percentage of cells in the G0/G1 phase from 72 to $61 \%(P<0.01)$. Taken together, SW treatment significantly increased the proliferation in HUVECs.

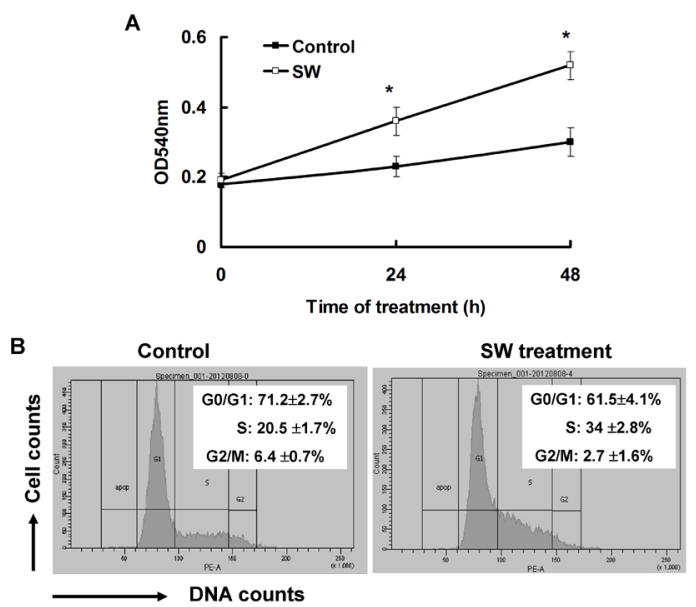

Figure 1. Shock wave (SW) treatment promotes cell proliferation in HUVECs. A. Following SW treatment, cell proliferation was assessed by MTT assay. The results are reported as means \pm standard deviations (SD) of four experiments. ${ }^{*} \mathrm{P}<0.05$ versus control (without $\mathrm{SW}$ treatment). B. Following $\mathrm{SW}$ treatment, the human umbilical vein endothelial cells (HUVECs) were collected and subjected to flow cytometric analysis. A typical experiment is shown. The results are reported as means \pm SD of four experiments. 


\section{SW treatment up-regulates the expression of VEGF.}

A previous study has reported that SW treatment increases the VEGF mRNA levels in cultured HUVECs (Nishida et al., 2004). Therefore, we examined the effect of SW treatment on the expression of VEGF in HUVECs, using qRT-PCR. SW treatment induced a 3.5-fold increase in the VEGF mRNA levels (Figure 2A). Meanwhile, western blotting revealed a marked increase in VEGF protein expression following the SW treatment (Figure 2B). It has been well-established that hypoxia-inducible factor 1 (HIF-1) regulates the expression of a variety of target genes, such as VEGF, in response to hypoxia (Gao et al., 2002). To investigate the role of HIF-1 in VEGF expression, the protein levels of HIF-1 $1 \alpha$, a subunit of HIF-1 that is regulated in response to hypoxia, was determined. Western blotting revealed that SW treatment did not affect the protein expression of HIF-1a (Figure 2C), suggesting that HIF-1 may not be involved in the SW treatment-induced expression of VEGF.
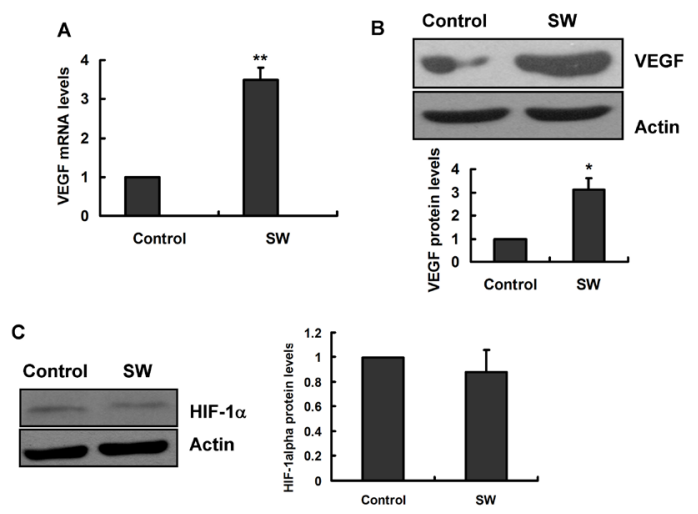

Figure 2. SW treatment induces the expression of vascular endothelial growth factor (VEGF) in HUVECs. A. Total RNA was extracted from SW-treated HUVECs, and subjected to real-time polymerase chain reaction (PCR). The mRNA levels of VEGF were standardized to the GAPDH levels. The results are reported as means \pm SD of four experiments. B. Protein levels of VEGF were detected by western blotting. The blot is typical of three independent experiments. The lower panel depicts the quantification of immunoreactivity levels. The data are reported as the percent change from the control. ${ }^{*} \mathrm{P}<0.05 ;{ }^{* *} \mathrm{P}<0.01$ with respect to the control (without SW treatment). C. Protein levels of HIF-1 $\alpha$ were detected by western blotting. The blot is indicative of three independent experiments. The right panel shows the quantification of immunoreactivity levels.

\section{Autocrine VEGF activates the ERK MAPK pathway}

The cell-culture medium was collected to determine the concentration of VEGF by ELISA. SW treatment resulted in a 2.5-fold increase in VEGF concentration in the cell culture medium (Figure 3A). VEGF has been shown to promote angiogenesis by activating multiple signal pathways, such as the ERK mitogen-activated protein kinase (MAPK) pathway (Zachary, 2003). Raf/MEK1/2/ERK signaling is required for VEGF-induced endothelial proliferation (Wu et al., 2000; Kim et al., 2010); therefore, we tested whether the SW treatment activated the ERK pathway. Post-SW treatment, we observed a significant increase in the phosphorylation of ERK; this was associated with its activation (Figure 3B). These results indicated the activation of the ERK MAPK pathway. However, pre-treatment with anti-VEGF neutralizing antibodies (and not isotype control antibodies) substantially suppressed the phosphorylation levels of ERK, suggesting that VEGF activates the ERK MAPK pathway in a cell-autonomous manner. 
A

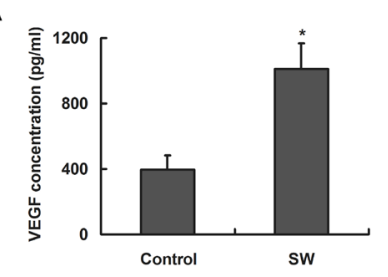

B
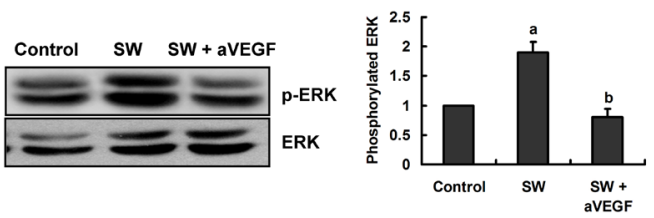

Figure 3. Autocrine VEGF induces activation of the extracellular signal-regulated kinase (ERK) signaling. A. Culture medium was collected following SW treatment. The VEGF levels were determined by enzyme-linked immunosorbent assay (ELISA). The results are reported as means $\pm S D$ of four experiments. ${ }^{*} P<0.05$ versus control (without $S W$ treatment). B. HUVECs pretreated with anti-VEGF neutralizing antibodies (aVEGF) were collected following SW treatment. The phosphorylated ERK levels were determined by western blot analysis. The blot is typical of three independent experiments. The right panel shows the quantification of immunoreactivity levels. ${ }^{\text {a }}<0.05$ versus control (without SW treatment); ${ }^{b} \mathrm{P}<0.05$ versus SW treatment.

\section{SW treatment-mediated endothelial proliferation is dependent on VEGF}

In order to investigate whether VEGF is responsible for SW treatment-mediated cell proliferation, HUVECs were treated with anti-VEGF neutralizing antibodies. As shown in Figure 4, the induction of cell proliferation by SW treatment (as assessed by MTT analysis) was significantly inhibited after the administration of anti-VEGF neutralizing antibodies, compared to treatment with isotype control antibodies. Furthermore, we tested the effect of ZM323881, a potent and selective VEGFR2/KDR inhibitor, on cell proliferation. The administration of ZM323881 (2 $\mu \mathrm{M})$ markedly abolished VEGF-stimulated cell proliferation in HUVECs. Finally, PD98059 (10 $\mu \mathrm{M})$, a specific inhibitor of MEK1/2 (Wu et al., 2000) was found to significantly block the SW treatment-stimulated cell proliferation in HUVECs (Figure 4). Taken together, this data reveal the involvement of VEGF in SW treatment-induced endothelial proliferation.



Figure 4. SW treatment promotes cell proliferation via the VEGF/ERK pathway in HUVECs. Following the SW treatment, the proliferation of HUVECs pretreated with anti-VEGF neutralizing antibodies (aVEGF), or ZM323881 (2 $\mu \mathrm{M})$, or PD98059 $(10 \mu \mathrm{M})$ was determined by the MTT assay. All results are reported as means \pm SD of three independent experiments. ${ }^{\mathrm{A} P}<0.05$ versus the control (without SW treatment); ${ }^{\mathrm{b}} \mathrm{P}<0.05$ versus cells subjected to the SW treatment. 


\section{DISCUSSION}

ESWT is an effective, safe, and non-invasive therapeutic strategy for severe coronary artery disease (Fukumoto et al., 2006; Khattab et al., 2007; Kikuchi et al., 2010; Vasyuk et al., 2010). In this study, we demonstrated that SW treatment up-regulates the expression of VEGF in HUVECs, leading to an increase in VEGF protein secretion. The autocrine VEGF in turn activates the ERK signaling in a cell-autonomous manner, leading to enhanced proliferation in HUVECs.

VEGF, a potential regulator of angiogenesis, induces endothelial cell proliferation, migration, and survival (Ferrara, 2002). VEGF binds to its receptor, VEGFR-2/KDR, and activates the Ras-dependent angiogenic signaling pathways, such as Raf-1/MEK1/2/ERK and p38 MAPK (Kim et al., 2010). The ERK signaling up-regulates the cyclin D1 expression and Rb phosphorylation, thereby promoting cell cycle progression. A previous study has demonstrated that SW treatment up-regulates VEGFR1/FIt1 mRNA levels in cultured HUVECs (Nishida et al., 2004). However, VEGFR2/KDR, and not VEGFR1/FIt1, has been shown to play a role in VEGF-mediated proliferation in endothelial cells (Wu et al., 2000; Mukhopadhyay et al., 2001; Zeng et al., 2003). Therefore, it is unlikely that the up-regulation of VEGFR1/FIt1 is responsible for SW treatmentinduced endothelial cell proliferation.

It has been shown that SW therapy can regulate the expression of many genes. In addition to VEGF, SW therapy up-regulates the expression of eNOS in the tendon-bone junction (Wang et al., 2003), and the ischemic skeletal muscle (Oi et al., 2008). Meanwhile, SW therapy induces the expression of a variety of anti-inflammatory cytokines, and suppresses the expression of proinflammatory cytokines after acute myocardial infarction (Abe et al., 2014). However, the molecular mechanisms by which SW treatment induces gene expression remain unclear. It is believed that shock waves raise the mechanotransduction of the tissue, to convert energy into biological signals (Wang et al., 2003). Although HIF-1 can up-regulate the expression of VEGF in response to hypoxia (Gao et al., 2002), our results suggest that HIF-1 is unlikely to be involved in SW treatment-induced VEGF expression. Likewise, our results also implicate that SW treatment itself does not induce a hypoxic environment in cell culture. Clearly, the precise mechanisms of SW therapy need to be elucidated in future studies.

\section{Conflicts of interest}

The authors declare no conflict of interest.

\section{ACKNOWLEDGMENTS}

Research supported by a grant (\#2012FB034) from the Joint Fund for the Yunnan Department of Science and Technology, and Kunming Medical University.

\section{REFERENCES}

Abe Y, Ito K, Hao K, Shindo T, et al. (2014). Extracorporeal low-energy shock-wave therapy exerts anti-inflammatory effects in a rat model of acute myocardial infarction. Circ. J. 78: 2915-2925.

Caro JJ, Migliaccio-Walle K, O'Brien JA, Nova W, et al. (2005). Economic implications of extended-release metoprolol succinate for heart failure in the MERIT-HF trial: a US perspective of the MERIT-HF trial. J. Card. Fail. 11: 647-656.

Ferrara N (2002). VEGF and the quest for tumour angiogenesis factors. Nat. Rev. Cancer 2: 795-803.

Fukumoto Y, Ito A, Uwatoku T, Matoba T, et al. (2006). Extracorporeal cardiac shock wave therapy ameliorates myocardial ischemia in patients with severe coronary artery disease. Coron. Artery Dis. 17: 63-70. 
Gao N, Ding M, Zheng JZ, Zhang Z, et al. (2002). Vanadate-induced expression of hypoxia-inducible factor 1 alpha and vascular endothelial growth factor through phosphatidylinositol 3-kinase/Akt pathway and reactive oxygen species. J. Biol. Chem. 277: 31963-31971.

Jessup M and Brozena S (2003). Heart failure. N. Engl. J. Med. 348: 2007-2018.

Khattab AA, Brodersen B, Schuermann-Kuchenbrandt D, Beurich H, et al. (2007). Extracorporeal cardiac shock wave therapy: first experience in the everyday practice for treatment of chronic refractory angina pectoris. Int. J. Cardiol. 121: 84-85.

Kikuchi Y, Ito K, Ito Y, Shiroto T, et al. (2010). Double-blind and placebo-controlled study of the effectiveness and safety of extracorporeal cardiac shock wave therapy for severe angina pectoris. Circ. J. 74: 589-591.

Kim CK, Choi YK, Lee H, Ha KS, et al. (2010). The farnesyltransferase inhibitor LB42708 suppresses vascular endothelial growth factor-induced angiogenesis by inhibiting ras-dependent mitogen-activated protein kinase and phosphatidylinositol 3-kinase/Akt signal pathways. Mol. Pharmacol. 78: 142-150.

Mukhopadhyay D, Sundereshan S, Rao C and Karande AA (2001). Placental protein 14 induces apoptosis in T cells but not in monocytes. J. Biol. Chem. 276: 28268-28273.

Nishida T, Shimokawa H, Oi K, Tatewaki H, et al. (2004). Extracorporeal cardiac shock wave therapy markedly ameliorates ischemia-induced myocardial dysfunction in pigs in vivo. Circulation 110: 3055-3061.

Oi K, Fukumoto Y, Ito K, Uwatoku T, et al. (2008). Extracorporeal shock wave therapy ameliorates hindlimb ischemia in rabbits. Tohoku J. Exp. Med. 214: 151-158.

Vasyuk YA, Hadzegova AB, Shkolnik EL, Kopeleva MV, et al. (2010). Initial clinical experience with extracorporeal shock wave therapy in treatment of ischemic heart failure. Congest. Heart Fail. 16: 226-230.

Vucenik I, Passaniti A, Vitolo MI, Tantivejkul K, et al. (2004). Anti-angiogenic activity of inositol hexaphosphate (IP6). Carcinogenesis 25: 2115-2123.

Wang CJ, Wang FS, Yang KD, Weng LH, et al. (2003). Shock wave therapy induces neovascularization at the tendon-bone junction. A study in rabbits. J. Orthop. Res. 21: 984-989.

Wu LW, Mayo LD, Dunbar JD, Kessler KM, et al. (2000). Utilization of distinct signaling pathways by receptors for vascular endothelial cell growth factor and other mitogens in the induction of endothelial cell proliferation. J. Biol. Chem. 275: 5096-5103.

Zachary I (2003). VEGF signalling: integration and multi-tasking in endothelial cell biology. Biochem. Soc. Trans. 31: 1171-1177.

Zeng H, Zhao D, Yang S, Datta K, et al. (2003). Heterotrimeric G alpha q/G alpha 11 proteins function upstream of vascular endothelial growth factor (VEGF) receptor-2 (KDR) phosphorylation in vascular permeability factor/VEGF signaling. J. Biol. Chem. 278: 20738-20745. 